\title{
TERAPIA DO CRESCIMENTO GENGIVAL INDUZIDO PELA CICLOSPORINA EM TRANSPLANTADOS RENAIS
}

\author{
Therapy in cyclosporine-induced gingival overgrowth in renal transplant patients
}

\begin{abstract}
Raphaela Bernardes Morais', Rafaela Wassoler Casagrande², Acir José Dirschnabel', Luciana Reis Azevedo', Sonia Mara Luczyszyn', Antonio Adilson Soares de Lima1, Maria Ângela Naval Machado'
\end{abstract}

\begin{abstract}
RESUMO
O crescimento gengival induzido por ciclosporina A em pacientes renais crônicos desenvolve-se no período entre um e três meses após o início da terapia. A extensão do aumento gengival varia desde leve mudança na margem gengival até a completa cobertura dos dentes, podendo interferir, quando severa, na mastigação, fonética e estética. O objetivo deste trabalho foi descrever dois casos clínicos de aumento gengival induzidos pela ciclosporina e suas respectivas terapias. No primeiro caso, optou-se por manter a saúde periodontal com controle de biofilme, e, no segundo caso, realizou-se a excisão cirúrgica e a terapia periodontal de suporte. Houve melhora do aspecto clínico em ambos os casos, pois ambas as terapias reduziram a inflamação. A terapia cirúrgica associada à escovação eliminou a fibrose e possibilitou a melhora na periodontite. Concluiu-se que o crescimento gengival foi reduzido após o controle profissional do biofilme dental ou após a combinação da técnica de higiene bucal associada à gengivectomia/gengivoplastia.
\end{abstract}

Descritores: Hiperplasia Gengival, Gengivite, Periodontite, Ciclosporina, Gengivectomia, Raspagem Dentária, Transplante Renal.

Instituição:

1. Clínica de Periodontia - Programa de Especialização em Odontologia - Pontifícia Universidade Católica do Paraná - Curitiba - Brasil

2. Serviço Odontológico - Fundação de Amparo à Pesquisa em Enfermidades Renais e Metabólicas - Pró-Renal - Curitiba - Brasil

Correspondência:

Profa Dra. Maria Ângela Naval Machado

Pontifícia Universidade Católica do Paraná - Programa de Pós-Graduação em Odontologia, Área de Estomatologia

Rua Imaculada Conceição, 1155 - Prado Velho

CEP 81611-970 - Curitiba - PR

Tel. 5541 3271-2592 - Fax 5541 3271-1405

E-mail: m.angela@pucpr.br

Recebido em: 18.08.2006

Aceito em: 27.09.2006

\section{INTRODUÇÃO}

O crescimento gengival (CG) representa uma resposta exacerbada do tecido gengival a uma variedade de condições locais e sistêmicas. ${ }^{1}$

Os transplantados renais ingerem medicações como ciclosporina A e nifedipina, que podem induzir ao $\mathrm{CG}^{2,3}$ As drogas indutoras de CG são divididas em três grupos principais que incluem os imunossupressores (ciclosporina A, prednisona, micofenolato mofetil e tacrolimus), os bloqueadores dos canais de cálcio (amlodipina, verapamil, diltiazina, nifedipina, oxidipina e felodipina) e os anticonvulsivantes (fenitoína). ${ }^{2,3}$

A incidência do CG oscila entre $25-81 \%$ nos transplantados renais. ${ }^{4}$ Sua patogenia está relacionada à interação de vários fatores, dentre os quais se destacam controle do biofilme, grau do crescimento gengival, inflamação gengival, extensão da destruição periodontal, dosagem, concentração plasmática, período de tempo do uso da CsA e idade do paciente..$^{3-5} \mathrm{O}$ biofilme dental atua como um fator irritante local e gera uma resposta hiperplásica no tecido gengival resultante da inflamação crônica persistente. ${ }^{2} \mathrm{O}$ objetivo deste trabalho foi descrever dois casos clínicos de pacientes transplantados renais com crescimento gengival induzido pela ciclosporina e suas respectivas terapias.

\section{CASO CLÍNICO I}

O paciente V.B. do sexo masculino, leucoderma, 21 anos de idade, realizava acompanhamento médico e odontológico na Fundação Pró-Renal de Curitiba-Paraná. A queixa principal era “crescimento da gengiva" e sangramento durante a escovação três meses pós-transplante. O paciente havia realizado transplante renal há aproximadamente dois anos. 
Os critérios para avaliação do crescimento gengival foram estabelecidos em graus, ${ }^{6}$ a seguir: Grau 0: nenhuma evidência clínica de crescimento gengival; Grau 1: crescimento gengival leve, cobrindo um terço da coroa dentária ou menos; Grau 2: crescimento gengival moderado; cobrindo mais de um terço da coroa dentária até sua metade; Grau 3: crescimento gengival severo, cobrindo dois terços da coroa dentária ou envolvendo completamente a gengiva inserida.

Ao exame clínico intrabucal, observou-se aumento gengival moderado (grau 2) na face vestibular dos dentes superiores, principalmente nas papilas interdentais dos dentes anteriores, estendendo-se para os posteriores (Figura 1). O crescimento gengival exibia coloração rósea nas papilas interdentais e áreas de eritema e ulceração na margem gengival (Figura 1). A gengiva apresentava-se edemaciada e de aspecto brilhante, e, em outras áreas, a consistência era firme, sugerindo fibrose (Figura 1). Observou-se sangramento à sondagem e presença de biofilme sobre a superfície dental. A radiografia periapical não revelou alterações periodontais significativas.

Foi realizado exame periodontal da profundidade de sondagem ou de bolsa que é definida como a distância da margem gengival até a base da bolsa ou sulco gengival. Essa profundidade é realizada por meio de inserção da sonda periodontal milimetrada no sulco gengival, e assim, é determinada a profundidade da bolsa tendo a margem gengival como ponto de referência até a base da bolsa ou sulco gengival. No presente caso, a profundidade média de sondagem variou entre 2 a $3,5 \mathrm{~mm}$, mas havia em algumas áreas a presença de pseudo-bolsas ou seja, de bolsa periodontal não verdadeira, causada pelo excesso de tecido gengival que recobre o dente. O diagnóstico foi de aumento gengival moderado associado à gengivite, ou seja, uns processos inflamatórios confinado à gengiva, que tem como características clínicas sangramento, eritema, edema, superfície brilhante e perda de adaptação da gengiva ao dente.

No tratamento, foi estabelecido inicialmente um programa de controle trimestral de biofilme. Esse programa consistiu na orientação sobre métodos de higiene bucal, uso de evidenciadores de biofilme, remoção de fatores de retenção de biofilme dental e profilaxia profissional. O índice de placa de O'Leary é um índice que avalia a ausência ou presença de biofilme sobre a superfície dental baseado no número de dentes e expresso em 0 a $100 \%{ }^{7}$ No início do tratamento, o índice de placa observado em dentes anteriores foi de $70 \%$, e ao final, foi reduzido para $30 \%$. Após três meses do controle profissional do biofilme, a gengiva, na região dos dentes anteriores superiores, apresentava aspecto lobulado, porém sem sinais de inflamação. $\mathrm{O}$ crescimento gengival que era moderado (grau 2), após a técnica de higiene bucal teve como resultado redução do volume, mostrando-se leve, de grau 1 (Figura 2). O acompanhamento clínico foi realizado durante 12 meses. Após esse período, o paciente foi encaminhado para realizar gengivoplastia, visando o restabelecimento funcional e estético.

\section{CASO CLÍNICO II}

Paciente A.A.S., 34 anos de idade, foi encaminhado do Hospital Cajuru da Pontifícia Universidade Católica do Paraná para tratamento na Clínica de Especialização em Periodontia da mesma instituição, relatando como queixa principal "crescimento da gengiva", sem saber definir com exatidão a data do início do crescimento. $\mathrm{O}$ paciente havia realizado o transplante renal há aproximadamente dois anos.

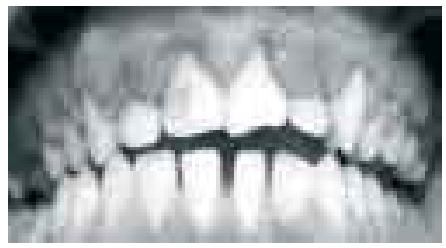

Figura 1 - Aspecto clínico inicial do CG.

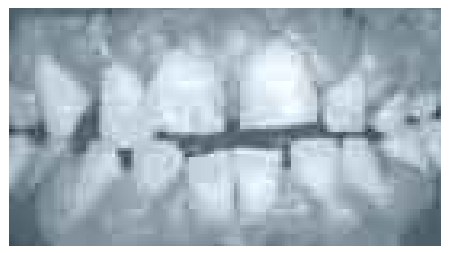

Figura 3 - Aspecto clínico inicial do $C G$.

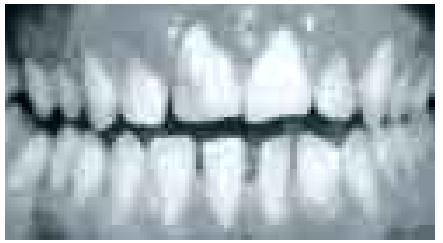

Figura 2 - Aspecto clínico final do $C G$.

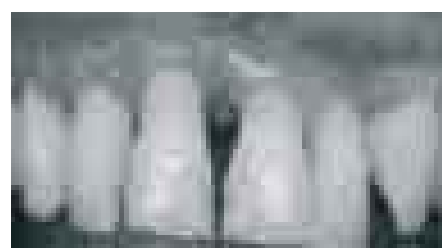

Figura 4 - Aspecto clínico do pósoperatório de 15 dias.
O exame clínico intrabucal revelou um aumento gengival moderado de grau 2 na face vestibular dos dentes anteriores superiores e inferiores (Figura 3). O aumento gengival era lobulado, principalmente na papila interdental, de consistência firme à palpação e coloração rósea (Figura 3). No exame clínico periodontal, a profundidade de sondagem média dos dentes anteriores superiores e inferiores variou entre 3,5 a $5,0 \mathrm{~mm}$, mostrando presença de bolsa periodontal, ou seja, um sulco gengival patológico localizado entre o dente e o epitélio sulcular limitado apicalmente pelo epitélio juncional, causando perda de inserção, destruição do osso alveolar e cemento radicular.

A presença de sangramento à sondagem também foi observada em algumas áreas. As radiografias panorâmicas e periapicais revelaram perda óssea. Esses exames comprovaram o diagnóstico de periodontite (processo patológico dos tecidos que suportam o dente, causando perda do ligamento periodontal, cemento radicular e destruição óssea alveolar). Portanto o paciente apresentava periodontite anterior ao transplante renal.

O tratamento proposto foi uma combinação da técnica de higiene bucal associada à excisão cirúrgica do CG (gengivectomia/ gengivoplastia).

Anteriormente à gengivectomia/gengivoplastia, o paciente recebeu tratamento periodontal que consistiu de raspagem e alisamento radicular, associado ao controle profissional do biofilme.

$\mathrm{Na}$ fase cirúrgica, foi realizada remoção das bolsas periodontais nos dentes anteriores superiores pela técnica clássica de gengivectomia/ gengivoplastia, pois a gengiva encontrava-se bem fibrosada e com superfície irregular (lobulada). No pós-operatório imediato, verificou-se remoção completa do tecido fibrótico e inflamatório, para se obter a regeneração tecidual satisfatória.

Após 15 dias do tratamento cirúrgico, observou-se cicatrização por primeira intenção, com formação de tecido queratinizado em área de mucosa gengival, com características clínicas de normalidade, porém com presença de recessão gengival devido à perda óssea existente no local e também devido à eliminação cirúrgica das bolsas periodontais (Figura 4). O crescimento gengival que era 
moderado (grau 2) após a terapia cirúrgica teve como resultado eliminação completa do CG (grau 0). O acompanhamento clínico foi realizado durante um mês após a cirurgia. Depois desse período, perdeu-se contato com o paciente, que mudou para outro estado.

\section{DISCUSSÃO}

$\mathrm{O}$ controle do biofilme, combinado à rigorosa manutenção profissional, leva freqüentemente à melhora do aspecto clínico do CG. ${ }^{8}$ Isto foi observado durante o tratamento para ambos os casos clínicos descritos.

De forma geral, a literatura demonstra que a inflamação gengival pré-existente é um fator que influencia na patogênese do CG induzido pelas drogas, e que a manutenção da higiene oral pode ser efetiva na sua prevenção e também nas recidivas. ${ }^{9}$ Entretanto, o controle do CG permanece um desafio, pois o CG pode persistir mesmo após rigoroso controle do biofilme dental, devido a alterações na morfologia gengival que dificultam o controle do biofilme e mantém a inflamação. ${ }^{10-12}$ Nestes casos, o tratamento cirúrgico é indicado para normalizar o contorno anatômico e funcional da gengiva. , $^{8,13}$

A inflamação gengival pode atuar como um fator potencializador do $\mathrm{CG}$, exacerbando o mesmo. ${ }^{14} \mathrm{O}$ indivíduo que não ingere CsA e tem péssima higiene, desenvolve gengivite, ou seja, uma inflamação localizada na gengiva, e não crescimento gengival, porém, a gengivite também causa alterações no volume gengival devido ao edema. Quando o transplantado ingere diariamente CsA e tem péssima higiene, pode vir a desenvolver aumento gengival, (desde que seja suscetível à medicação), somado à inflamação gengival pré-existente. A CsA pode atuar como um indutor do CG em indivíduos suscetíveis. ${ }^{12,15}$

O biofilme (antigamente chamado de placa dental bacteriana) tem papel fundamental no início e na gravidade do crescimento gengival. Alguns autores ${ }^{16-19}$ encontraram uma correlação significativa entre o biofilme ou a inflamação gengival e o início ou gravidade do aumento gengival. Entretanto, outro autor ${ }^{20}$ sugeriu que, nem o biofilme nem a inflamação, influenciam a incidência do aumento gengival. $\mathrm{O}$ fato é que devido a alterações na morfologia gengival, existe uma dificuldade no controle do biofilme e há uma correlação significativa entre a profundidade da bolsa e o aumento gengival ${ }^{15}$. O aumento gengival leva à formação de pseudo-bolsas, com profundidade de sondagem maior ou igual a $3 \mathrm{~mm}$, porém nem sempre existe uma relação com os índices de placa e gengival, ou seja, com a higiene bucal. ${ }^{20}$

A patogênese do CG é multifatorial, e os principais fatores envolvidos são as drogas, a inflamação causada pelo biofilme, a suscetibilidade dos fibroblastos gengivais e fatores genéticos. ${ }^{16}$

A substituição da medicação seria uma opção terapêutica viável na tentativa de controlar o CG, porém em transplantados renais nem sempre existe essa flexibilidade. ${ }^{3}$ A CsA poderia ser substituída pelo tacrolimus, que não induz ao $\mathrm{CG}^{12}$, porém esta medicação tem um alto custo. Quando não é possível a substituição da medicação ou o CG não regride o suficiente para permitir um controle adequado do biofilme dental, indica-se a eliminação do CG por técnicas

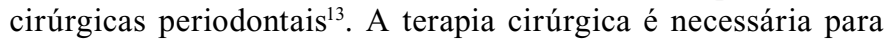
restabelecer a estética e a função do periodonto, facilitando a higiene bucal. ${ }^{13}$

A excisão cirúrgica não é um tratamento definitivo para o crescimento gengival. Medidas de higiene bucal profissional deverão ser realizadas periodicamente ${ }^{8}$. Além disso, pacientes imunossuprimidos não deverão ser submetidos a extensas cirurgias periodontais regularmente. Para não agravar a condição sistêmica, a manutenção da higiene bucal é fundamental para prolongar a recidiva do CG.

A recidiva do CG induzido por drogas após terapia cirúrgica é esperada, embora não exista previsibilidade em longo prazo do grau e da gravidade do CG. ${ }^{16}$

Nos dois relatos de caso acima descritos, observamos que o controle do biofilme pelo próprio paciente nem sempre é satisfatório, e que a medicação tem ação contínua na gengiva. $\mathrm{O}$ desestímulo em realizar terapia periodontal de suporte, ou seja, retorno periódico ao consultório odontológico para avaliar o CG e controlar a higiene bucal é comum nos transplantados renais. Isto pode ocorrer em função da gravidade da condição sistêmica, que os obriga a tratamentos médicos freqüentes e prolongados durante suas vidas. ${ }^{21}$ Outro aspecto é a repercussão pelos pacientes companheiros ou pela própria equipe médica de que o crescimento gengival é inevitável e não tratável. ${ }^{21}$ Embora esses pacientes tenham consciência das alterações gengivais, metade desses indivíduos acredita necessitar de tratamento, e apenas uma minoria está preocupada com a aparência gengival. ${ }^{21}$ Outros fatores que o presente artigo poderia sugerir e que podem influenciar o desestímulo ao tratamento periodontal seriam fatores psicológicos, sociais e econômicos.

\section{CONCLUSÕES}

Baseados nos casos clínicos acima relatados podemos concluir que o crescimento gengival foi reduzido após controle profissional do biofilme dental ou após combinação da técnica de higiene bucal associada à gengivectomia/gengivoplastia.

Uma melhor higiene bucal e a remoção de fatores que retêm o biofilme melhoraram a saúde gengival, mas estas medidas falham em prevenir o desenvolvimento ou a recorrência do crescimento gengival em pacientes suscetíveis.

\section{AGRADECIMENTOS}

Fundação de Amparo à Pesquisa em Enfermidades Renais e Metabólicas - Pró-Renal, Curitiba-PR.

Clínica de Periodontia - Programa de Especialização em Odontologia - Pontifícia Universidade Católica do Paraná - Curitiba-PR.

\section{ABSTRACT}

Cyclosporine A-induced gingival overgrowth in kidney transplant patients usually appears one to three months after the initial therapy. The severity of the gingival overgrowth varies from mild changes of the gingival margin up to the totally covered dental crown, causing esthetic and functional problems. The purpose of this paper is to relate two clinical cases of gingival enlargement and the respective therapy. In the 
first case, the periodontal health was maintained with professional biofilm control. The second case was treated for surgical excision, followed by a periodontal support therapy. The clinical aspect of the CG in both cases improved, since therapies reduced the inflammation. The surgical therapy associated to biofilm control was able to eliminate fibrosis, improving the periodontitis condition. Concluding, our findings suggest that the gingival overgrowth was reduced after biofilm dental control or after combining it with gingivectomy/gingivoplasty.

Keywords: Gingival Hyperplasia, Gingivitis, Periodontitis, Cyclosporine, Gingivectomy, Dental Scaling, Kidney Transplantation.

\section{REFERENCES}

1. Seymour RA. Drug-induced overgrowth. Adverse Drug React Toxicol Rev. 1993;12:215-32.

2. Tavassoli S, Yamalik N, Çaglayan F, Eratalay K. The clinical effects of nifedipina on periodontal status. J Periodontol. 1998;69:108-12.

3. Daley TD, Wysocki GP, Day C. Clinical and farmacologic correlations in cyclosporine-induced gingival hyperplasia. Oral Surg Oral Med Oral Pathol. 1986; $62: 417-421$.

4. Seymour RA, Jacobs DJ. Ciclosporin and the gingival tissues. J Clin Periodontol. 1992;19:1-11.

5. Laupacis A, Keown PA, Ulan RA, McKenzie N, Stiller CR. Ciclosporin A: a powerful immunosuppressant. Can Med Assoc J. 1982;6:1041-6.

6. Angelopoulos AP, Goaz PW. Incidence of diphenylhydantoin gingival hyperplasia. Oral Surg Oral Med Oral Pathol. 1972;34:898-906.

7. Rodrigues JA, dos Santos PA, Garcia PP, Corona SA, Loffredo LC. Evaluation of motivation methods used to obtain appropriate oral hygiene levels in schoolchildren. Int J Dent Hyg. 2003;1:227-32.

8. Daley TD, Wisocki GP. Cyclosporine Therapy. Its significance to the periodontist. J Periodontol. 1984;12:708-712.

9. Pilloni A, Camargo PM, Carere M, Carranza Jr FA. Surgical treatment of ciclosporine A- and nifedipine-induced gingival enlargement: gingivectomy versus periodontal flap. J Periodontol. 1998;69:791-797.

10. Ferguson CA, Whyman RA. Dental management of people with renal disease and renal transplants. N Z Dent J. 1998;94:125-130.

11. Prabhau A, Mehta DS. A morphologic comparison of gingival changes influenced by cyclosporin and tacrolimus in rats: an experimental study. J Periodontol. 2006;77:265-70
12. Radwan-Ocko M, Boratynska M, Klinger M, Zietk M. Risk factors of gingival overgrowth in kidney transplant recipients treated with cyclosporine. Ann Transplant. 2003;8:57-62

13. Newman MG, Takei HH, Carranza FA. Carranza Periodontia Clínica. Rio de Janeiro: Guanabara Koogan;2004.

14. De Oliveira CF, Diniz FS, de Miranda CLO, Da Costa JE, Aguiar MA. Prevalence, severity, and risk variables associated with gingival overgrowth in renal transplant subjects treated under tacrolimus or cyclosporin regimens. J Periodontol. 2006;77:969-75.

15. Margiota V, Pizzo I, Pizzo G, Bárbaro A. Cyclosporin- and nifedipine- induced gingival overgrowth in renal transplant patientes: correlations with periodontal and pharmacological parameters, and HLA-antigens. J Oral Pathol Med. 1996;25:128-34.

16. Seymour RA, Thomason JM, Nolan A. Oral lesions in organ transplant patients. J Oral Pathol Med. 1997;26:297-304.

17. Thomason JM, Seymour RA, Rice N. The prevalence and severity of cyclosporin and nifedipine induced gengival overgrowth. J Clin Periodontol. 1993;20:37-40.

18. Somacarrera ML, Hérnandez G, Acro J, Moskow, BS. Factors related to the incidence and severity of cyclosporine - induced gingival overgrowth in transplant patients. A longitudinal study. J. Periodontol. 1994,65:671-675.

19. Pernu HE, Pernu H, Huttunen KRH, Nieminen PA, Knuutila MLE. Gingival overgrowth among renal transplant recipients related to immunosuppressive medication and possible local background factors. J Periodontol 1992;63:548-53.

20. Seymour RA, Smith DG. The effect of a plaque control programme on the incidence and severity of cyclosporin induced gingival changes. J Clin Periodontol. 1991;18:107-110.

21. Spratt H, Boomer S, Irwin CR, Marley JJ, James JA, Maxwell P, Middleton D, Linden GJ. Cyclosporin associated gingival overgrowth in renal transplant recipients. Oral Dis. 1999;5:27-31. 